\title{
KRAS (G12D) Cooperates with AML1/ETO to Initiate a Mouse Model Mimicking Human Acute Myeloid Leukemia
}

\author{
Shanmin Zhao a,d Yuxia Zhang ${ }^{b, d}$ Kun Shab ${ }^{b, d}$ Qiu Tanga,d Xiaohua Yang ${ }^{c}$ \\ Chenlin Yu ${ }^{a}$ Zhixue Liu ${ }^{a}$ Wei Sun ${ }^{a}$ Liping Cai ${ }^{a}$ Chen Xua Shufang Cuia \\ aLaboratory Animal Center of Second Military Medical University, Shanghai, 'bivision of Educational \\ Support, Training Department of Second Military Medical University, Shanghai, 'Shanghai Chest \\ Hospital affiliated Shanghai Jiaotong University, Shanghai, China; ${ }^{\mathrm{d} T h e s e}$ authors contributed equally to \\ this work ('First Author: S. Zhao; Y. Zhang; K. Sha; Q. Tang)
}

\section{Key Words}

Ras mutations • Acute myeloid leukemia • Leukemogenesis • K-ras • AML1/ETO

\begin{abstract}
Background/Aims: It has been demonstrated that KRAS mutations represent about $90 \%$ of cancer-associated mutations, and that KRAS mutations play an essential role in neoplastic transformation. Cancer-associated RAS mutations occur frequently in acute myeloid leukemia (AML), suggesting a functional role for Ras in leukemogenesis. Methods: We successfully established a mouse model of human leukemia by transplanting bone marrow cells cotransfected with the K-ras (G12D) mutation and AML1/ETO fusion protein. Results: Mice transplanted with AML/ETO+KRAS co-transduced cells had the highest mortality rate than mice transplanted with AML/ETO- or KRAS-transduced cells (115d vs. 150d). Upon reaching a terminal disease stage, EGFP-positive cells dominated their spleen, lymph nodes, peripheral blood and central nervous system tissue. Immunophenotyping, cytologic analyses revealed that AML/ETO+KRAS leukemias predominantly contained immature myeloid precursors $\left(\mathrm{EGFP}^{+} / \mathrm{C}-\mathrm{Kit}^{+} / \mathrm{Mac}-1^{-} / \mathrm{Gr}^{-} \mathbf{1}^{-}\right)$. Histologic analyses revealed that massive leukemic infiltrations were closely packed in dense sheets that effaced the normal architecture of spleen and thymus in mice transplanted with AML1/ETO + KRAS co-transduced cells. K-ras mRNA and protein expression were upregulated in bone marrow cells of the K-ras group and AML1/ ETO + Kras group. The phosphorylation of MEK/ERK was significantly enhanced in the AML1/ ETO + Kras group. The similar results of the AML1/ETO + Nras group were consistent with those reported previously. Conclusion: Co-transduction of Kras ${ }^{G 12 D}$ and AML1/ETO induces acute monoblastic leukemia. Since expression of mutant K-ras alone was insufficient to induce leukemia, this model may be useful for investigating the multi-step leukemogenesis model of human leukemia.
\end{abstract}

Copyright $\odot 2014$ S. Karger AG, Basel 


\section{Cellular Physiology and Biochemistry}

Cell Physiol Biochem 2014;33:78-87

DOI: 10.1159/000356651

Published online: January 17, 2014

C) 2014 S. Karger AG, Basel

www.karger.com/cpb

\section{Introduction}

Of the three genes in the RAS family (KRAS, NRAS and HRAS), KRAS is the most frequently mutated in human tumors, with an incidence of $70-90 \%$ in pancreatic adenocarcinoma, $50 \%$ in colon adenocarcinoma, $25-50 \%$ in lung adenocarcinoma, and approximately $20 \%$ in other types of human cancer, including carcinomas of the lung, colon, and pancreas [17]. RAS oncogene mutations, including KRAS (G12D), are also observed in approximately one third of patients with human malignancies $[8,9]$. When mutated at codon 12,13 or 61 , the K-ras mutant proteins remain active and can constitutively transduce signals through the mitogen-activated protein kinase (MAPK) pathway and the phosphoinositide-3-kinase (PI3K) pathway [10-13]. It has been demonstrated that KRAS mutations represent about $90 \%$ of cancer-associated mutations, and that KRAS mutations play an essential role in neoplastic transformation. Cancer-associated RAS mutations, which introduce amino acid substitutions at codons 12,13 , or 61 , result in oncogenic Ras proteins that accumulate in the active, GTPbound conformation due to defective guanine nucleotide hydrolysis $[9,14]$. Several animal models have been generated to study the role of KRAS mutations in tumorigenesis [1,15-17].

The $t(8 ; 21)(q 22 ; q 22)$ translocation is one of the most common structural aberrations observed in acute myelogenous leukemia (AML), and can be found in 5-12\% of AML cases and in one third of karyotypically abnormal cases of AML with maturation. The t(8;21)(q22;q22) translocation generates the AML1/ETO oncogenic transcription factor which initiates acute myeloid leukemia by recruiting co-repressor complexes to DNA [18]. This observation alone is difficult to reconcile with leukemogenesis, as growth arrest would not be favorable for the propagation of cells harboring the $t(8 ; 21)$ translocation [19]. The full-length AML1-ETO fusion protein, resulting from the $t(8 ; 21)$ ( $222 ; \mathrm{q} 22)$ translocation in human acute myeloid leukemia (AML), is not sufficient to induce leukemia in animal models [20-22], suggesting that additional mutations are required for leukemogenesis. Several studies have shown that anti-differentiation and pro-proliferative genes cooperate to promote AML in mice [23-25]. The existence of RAS mutations in diverse myeloid malignancies raises the possibility that they invariably represent secondary events that cooperate with a spectrum of initiating genetic lesions. NRAS (G12D) cooperates with AML1/ET09a or MLL/ENL to accelerate the onset and reduce overall survival in leukemia [25]. To test our hypothesis that an activating KRAS (G12D) mutation cooperates with AML1-ETO to induce overt AML, we generated a murine transduction and transplantation model with both the KRAS (G12D) mutation and AML1-ETO translocation.

\section{Materials and Methods}

\section{Ethics statement}

Six- to eight-week-old male C57BL/6J mice were purchased from Shanghai Laboratory Animal Center, Chinese Academy of Sciences (Shanghai, China). The animal handling and procedures in this study were in accordance with the current Chinese regulation "GB 14925-2010 Laboratory animal requirements of environment and housing facilities" (Chinese version). The complete protocol was reviewed and approved by the Institutional Animal Care and Use Committee of the Second Military Medical University. All surgery was performed under sodium pentobarbital anesthesia, and all efforts were made to minimize suffering.

Construction of retrovirus vectors

Total RNA was isolated from the peripheral blood mononuclear cells of leukemia patients using ISOGEN-LS (Nippon Gene Co, Tokyo, Japan) according to the manufacturer's protocol. Firststrand complementary DNA (cDNA) was synthesized by reverse transcription using the RNA as the template. The $K R A S^{\mathrm{G} 12 \mathrm{D}}$ gene mutation was amplified from the cDNA using the primers: 5' - CTGGCTAGCATGACTGAATATAAACTTGTG GTAGTTGGAG CT GATGGCGTAG GCAAGAGTGCCTT-3' (forward) and 5'-ACAGCGGCCGCTTACATAATTACACACTTTGTCTTTGACTTCTTTT-3' (reverse); the $N R A S^{\mathrm{G} 12 \mathrm{D}}$ gene mutation was amplified using the primers: 


\section{Cellular Physiology and Biochemistry}

Cell Physiol Biochem 2014;33:78-87

\begin{tabular}{l|l}
\hline DOI: $10.1159 / 000356651$ & (C) 2014 S. Karger AG, Basel
\end{tabular}

Published onlıne: January 17, $2014 \quad$ www.karger.com/cpb

Zhao et al.: Development of a Mouse Model Mimicking Human AML

5'-TGGGCTAGCATGACTGAGTACAAACTGGTGGTGGTTGGAGCAGATGGTGTTGGGAAAAGCGCACTG-3' (forward); 5'-AAAGCGGCCGCTTACATCACCACACATGGCAATCCC-3' (reverse). The PCR products were analyzed by $1 \%(\mathrm{w} / \mathrm{v}$ ) agarose gel electrophoresis ( $60 \mathrm{~min}$ at $100 \mathrm{~V}$ in TAE $1 \times$ electrophoresis buffer) and visualized using ethidium bromide (BioRad, Hercules, CA, USA) and photographed under UV light. The amplified fragments were excised, purified, and digested using the restriction enzymes Nhe I and Not I and cloned separately into the Nhe I and Not I sites of the vector pCDH-CMV-MCS-EF1-copGFP (System Biosciences, Mountain View, CA, USA). The recombinant vectors were named pCDH-K-ras ${ }^{\mathrm{G} 12 \mathrm{D}}$ and $\mathrm{pCDH}-\mathrm{N}$ ras $^{\mathrm{G} 12 \mathrm{D}}$, respectively. The AML1/ETO fusion gene was amplified by PCR as described previously [26] and subcloned into the vector pCDH-CMV-MCS-EF1-copGFP by double digestion with Xba I and Not I. The recombinant vector was named pCDH-AML1/ETO.

\section{Viral production and titer analysis}

293T cells were plated into $25 \mathrm{~cm}^{2}$ tissue culture dishes at a density of $1 \times 10^{6}$ cells/flask and transfected with $1 \mu \mathrm{g}$ of the retroviral vectors (pCDH-K-ras ${ }^{\mathrm{G} 12 \mathrm{D}}, \mathrm{pCDH}-\mathrm{N}-\mathrm{ras}^{\mathrm{G} 12 \mathrm{D}}$ or $\mathrm{pCDH}-\mathrm{AML1} / \mathrm{ETO}$, respectively) using $2.5 \mu$ FuGENE HD transfection reagent (Promega, Madison, WI, USA) according to the manufacturer's instructions. Retroviral supernatant was harvested $48 \mathrm{~h}$ after transfection. To assay for viruses carrying the selectable marker enhanced green fluorescent protein (EGFP), recipient 293T cells were seeded at 5 $\times 10^{5}$ per $60 \mathrm{~mm}$ dish on day one. On day 2 , the medium was changed to medium containing $2 \mu \mathrm{g} / \mathrm{mL}$ polybrene and $2 \%$ fetal bovine serum (FBS), and various amounts of test virus were added. On day 3 , the virus-containing medium was removed, and the cells were incubated in complete medium. Five days later, the viral titers were determined by counting the number of EGFP-positive cells per well using a fluorescence microscope. Titers were determined in triplicate wells by counting five fields per well and expressed as transducing units $/ \mathrm{mL}(\mathrm{TU} / \mathrm{mL}) \pm$ standard deviation.

\section{Cell transplantation pilot study}

For the pilot study, six- to eight-week-old male C57BL/6J mice were provided with drinking water containing antibiotics for 1 week, and were then irradiated with X-rays at 8 Gy or 10 Gy ( 40 per group). Bone marrow cells were harvested from the long bones of male C57BL/6J mice and cultured in IMDM medium (GIBCO, Carlsbad, CA, USA) supplemented with 10\% FBS and penicillin/streptomycin (50 U/mL). Cultured untransfected mouse bone marrow cells $\left(0.5-1.0 \times 10^{6}\right.$ cells in PBS) were injected into the tail vein of the irradiated mice within $24 \mathrm{~h}$ after irradiation. The control group received only normal saline after irradiation (10 per group). The survival time of the mice was observed; 8 Gy led to better survival compared to mice irradiated with 10 Gy (data not shown).

Bone marrow transfection and transplantation model

To transplant transfected bone marrow cells, the bone marrow cells of six- to eight-week-old male C57BL/6J mice were harvested as described as above, randomly divided into four groups (20 mice per group), placed into non-tissue culture-treated 6-well plates (Becton Dickinson) and then coated with 2 $\mathrm{ml}$ of different combinations of viral suspension as follows: (1) NRAS ${ }^{\mathrm{G} 12 \mathrm{D}}$; (2) KRAS ${ }^{\mathrm{G} 12 \mathrm{D}}$; (3) AML1/ETO; (4) $A M L 1 / E T O+K R A S^{\mathrm{G} 12 \mathrm{D}}$; or (5) $A M L 1 / E T O+N R A S^{\mathrm{G} 12 \mathrm{D}}$, at a multiplicity of $40 \mathrm{PFU}$ per cell for $6 \mathrm{~h}$ at $37^{\circ} \mathrm{C}$. After $6 \mathrm{~h}$, the bone marrow cells were harvested, washed twice with phosphate-buffered saline and used for transplantation. About $1 \times 10^{6}$ cells were injected into mice of the relevant groups. The remaining uninfected cells were examined using a fluorescence activated cell sorter (FACS) after $48 \mathrm{~h}$ of culture to determine the infection efficiency. All the transplanted mice were irradiated with X-rays at 8 Gy after drinking water containing antibiotics for one week; the mice were injected with the cells within $24 \mathrm{~h}$ after irradiation. Survival was evaluated 150 days after bone marrow cell transplantation.

\section{Reverse Transcription-PCR (RT-PCR)}

Total RNA was isolated from the bone marrow cells of transplanted mice using TRIzol Reagent (Invitrogen, Carlsbad, CA, USA) at 8 weeks after transplantation. The concentration of total RNA was measured using a NanoDrop 1000 (Nanodrop Technologies Inc., Wilmington, DE, USA), and first-strand cDNA was synthesized using Superscript II (Invitrogen) with an oligo (dT) primer. KRAS, NRAS, AML1-ETO or $\beta$-actin were amplified with specific primers using Ex-Taq polymerase (Takara). The primer sets were as follows: KRAS: 5'-GTAGTTGGAGCTGATGGCGTAG-3' (forward) and 5'-CACAAAGAAAGCCCTCCCCA-3' 


\section{Cellular Physiology and Biochemistry}

Cell Physiol Biochem 2014;33:78-87

DOI: 10.1159/000356651

Published onlıne: January 17, 2014

(C) 2014 S. Karger AG, Basel

www.karger.com/cpb

81

Zhao et al.: Development of a Mouse Model Mimicking Human AML
Fig. 1. Schematic diagram of the retroviral vectors and confirmation of the ability of the retroviral vectors to infect human bone marrow cells. (A) MSCVbased retroviral constructs were used to express K-Ras (G12D), N-Ras (G12D) and AML1/ETO with the fluorescent marker enhanced green fluorescence protein (EGFP). (B) Immunofluorescence of human bone marrow cells transduced with the indicated viral vectors demonstrated bright, cytosolic enhanced green fluorescence protein (EGFP) expression.

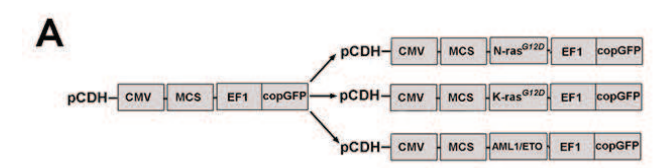

B

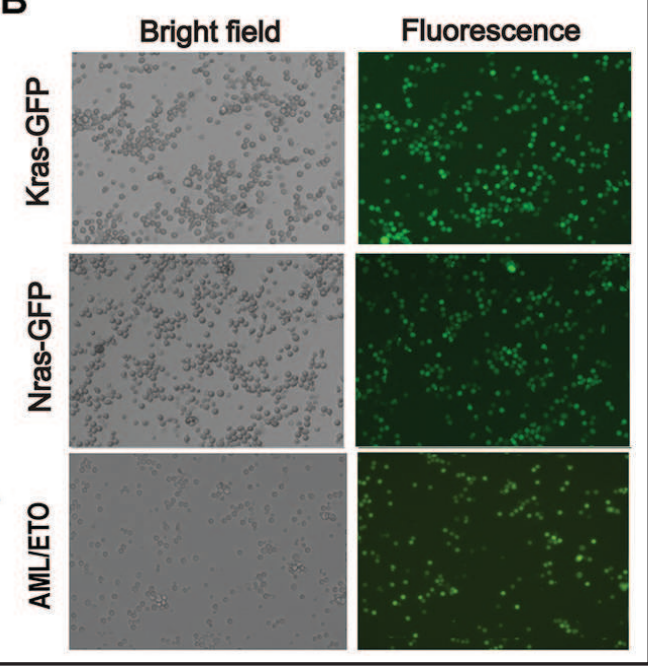

(reverse); NRAS: 5'-TGGTTGGAGCAGATGGTGTTG-3' (forward) and 5'-AAGCCTTCGCCTGTCCTCAT-3' (reverse); AML1-ETO: 5'-CAGGGCAGCCACAGCAACT-3' (forward) and 5'-AGAATGGCTCGTGCCATTAGTT-3' (reverse); $\beta$-actin: 5'-GGCTGTATTCCCCTCCATCG-3' (forward) and 5'-CCAGTTGGTAACAATGCCATGT T-3' (reverse).

\section{Flow Cytometric Analysis}

Single-cell suspensions were prepared from the spleen, lymph nodes, peripheral blood and central nervous system (CNS) of the mice at a terminal disease stage (severe leukocytosis in peripheral blood smears, moribund appearance). The tissues were minced through a metal mesh, and the bone marrow (BM) was flushed from the tibiae and femurs with IMBM containing 10\% FBS using a 27-gauge needle. Surface markers were detected using the following fluorescent human specific antibodies: allophycocyanin (APC) anti-mouse Ly-6G (Gr-1, Gr1); APC anti-mouse CD11b (Integrin alpha M, Mac-1 alpha); and APC anti-mouse CD117 (c-Kit, cKit), all of which were obtained from Packard Bioscience Company, Toronto, Ontario, Canada. The cells were stained with the relevant antibodies for 15 min on ice, washed with cold PBS containing $2 \%$ FCS, and stained with the appropriate secondary antibodies (if necessary). After a final wash, the cells were subjected to flow cytometric analysis using a FACSCanto II cytometer (BD Biosciences). To distinguish between the EGFP $(510 \mathrm{~nm})$ and Venus $(535 \mathrm{~nm})$ fluorescence, an appropriate combination of mirrors and optical filters was used. The proportion of each lineage was calculated using the FACS Diva software (BD Biosciences).

\section{Blood smears and histology}

Peripheral blood smears and bone marrow cytospins were stained with Wright-Giemsa stain (Sigma). Tissue specimens for histology were preserved in cold $4 \%$ formaldehyde in phosphate-buffered saline (PBS). Embedding, sectioning and staining with hematoxylin and eosin (Sigma) was performed using standard protocols.

\section{Western blot analysis}

Cell lysates were prepared by suspending the cells in ice-cold lysis buffer, boiled for 5 min, separated by SDS-PAGE, and transferred to PVDF membranes (Millipore, Billerica, MA, USA), blotted for $1 \mathrm{~h}$ with primary antibodies against N-ras (Santa Cruz Biotech), K-ras (Santa Cruz Biotech), AML1-ETO (Santa Cruz Biotech), Pan-ras (Upstate Biotechnologies), MEK and ERK (Santa Cruz Biotech), p-MEK and p-ERK (Cell 
Fig. 2. $K R A S$ mutants with AML1/ETO-induced acute myeloid leukemia in mice. (A) Kaplan-Meier survival curves for mice transplanted with bone marrow cells transduced with the indicated retroviral constructs $(n=40$ mice per group). (B) Fluorescence-activated cell sorting analysis of the cells from the spleen, lymph nodes, peripheral blood and central nervous system tissue of mice transduced with the indicated retroviral constructs.
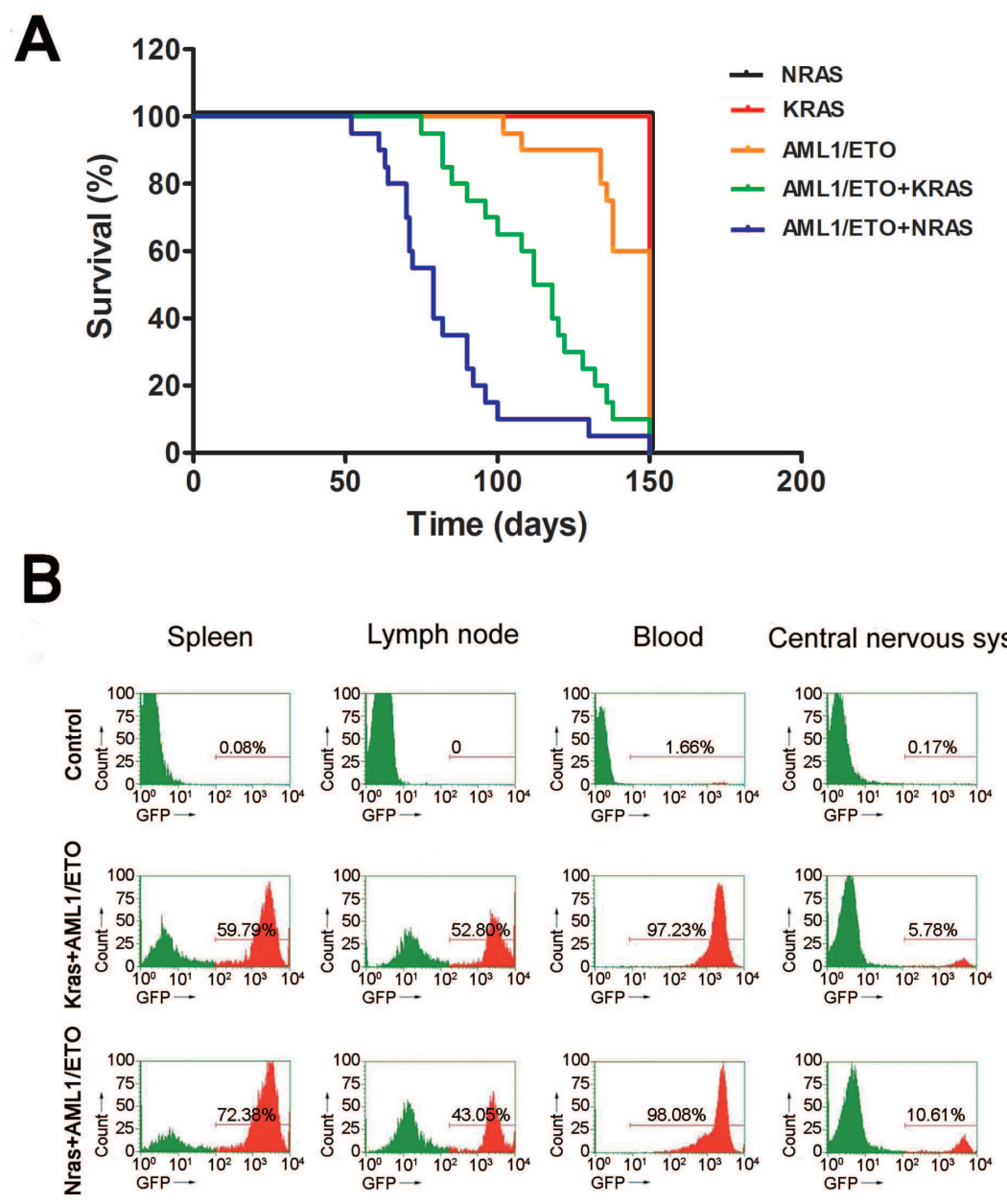

Signaling Technology) and $\beta$-actin (Santa Cruz Biotech). The blots were washed three times with $10 \mathrm{mM}$ Tris (pH 7.4), 0.1\% Tween-20, and $100 \mathrm{mM} \mathrm{NaCl}$, incubated for $1 \mathrm{~h}$ with secondary antibodies conjugated to horseradish peroxidase and washed three times. Blots were developed using the ECL detection system (Pierce, Rockford, IL, USA).

\section{Results}

Construction of retroviral vectors

In order to create retroviral vectors to express fusion proteins of K-ras ${ }^{\mathrm{G} 12 \mathrm{D}}$ or N-ras ${ }^{\mathrm{G} 12 \mathrm{D}}$, the $K R A S^{G 12 D}$ and $N R A S^{G 12 D}$ genes were cloned. The sequences of the resulting PCR products (data not shown) were similar to that reported in GenBank (KRAS, NM_004985 and NRAS, NM_002524). The PCR products were then cloned into the vector pCDH-CMV-MCSEF1-copGFP and the positive recombinant retroviral vectors were confirmed by PCR and sequencing (data not shown). K-ras ${ }^{G 12 D}$ and N-ras ${ }^{G 12 D}$ were coexpressed with enhanced green fluorescent protein (EGFP) from these bicistronic constructs (Fig. 1A). Due to the high fluorescence signals, bright EGFP expression could easily be detected under a standard fluorescence microscope in bone marrow cells transfected with the recombinant retrovirus vectors expressing $K R A S^{\mathrm{G} 12 \mathrm{D}}, N R A S^{\mathrm{G} 12 \mathrm{D}}$ or AML1-ETO (Fig. 1B).

The recombinant vectors (pCDH-KRAS ${ }^{G 12 D}$ and pCDH-NRAS ${ }^{G 12 D}$ ), which encode the reporter gene EGFP, were transiently transfected into 293T cells. The transfected cells 


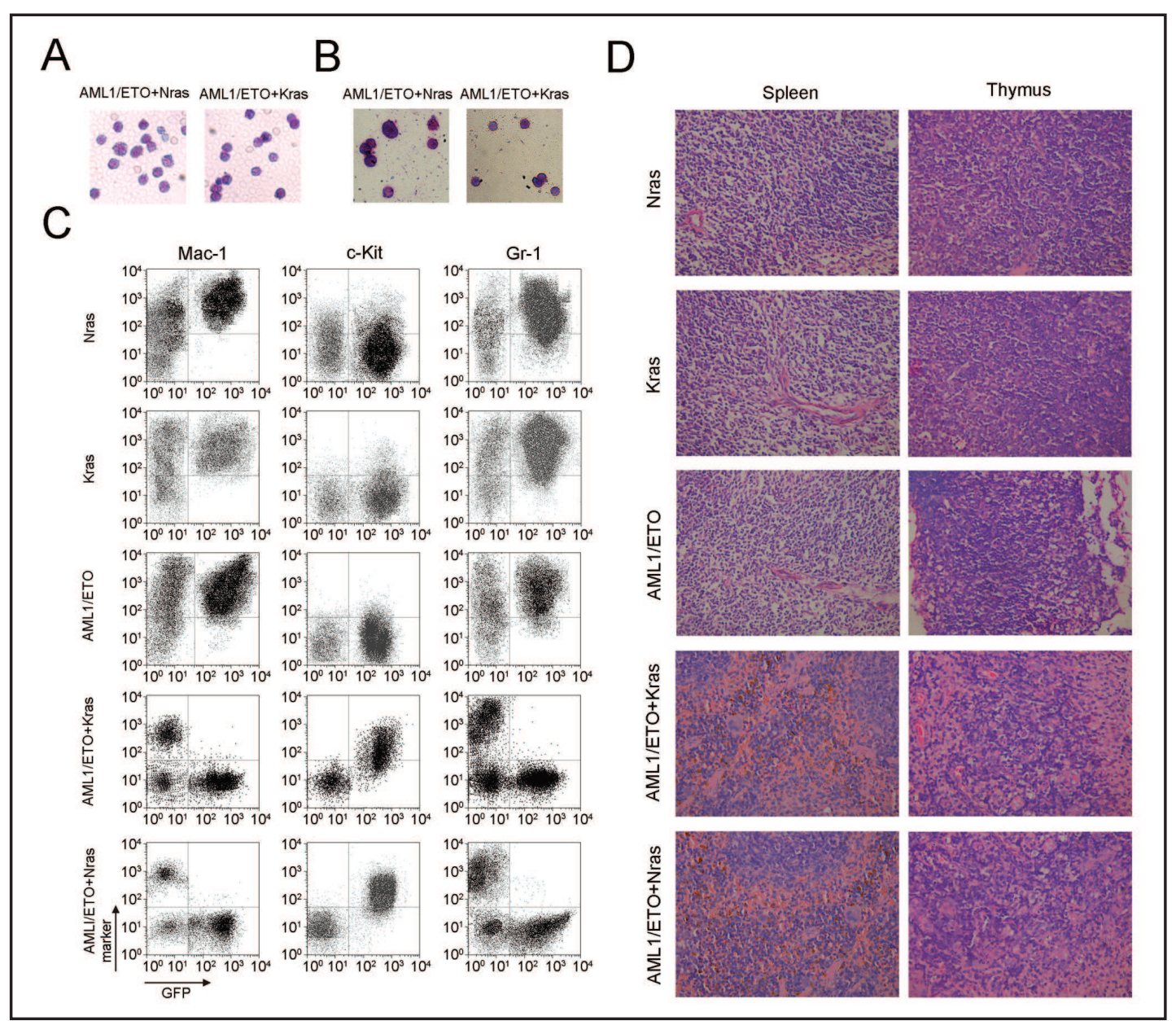

Fig. 3. Characterization of primary leukemias in AML1/ETO+KRAS ${ }^{G 12 D}$ mice. (A) Peripheral blood cells and (B) bone marrow cells of representative moribund AML1/ETO+KRAS ${ }^{G 12 D} /$ AML1/ETO+NRAS ${ }^{G 12 D}$ mice observed by Wright-Giemsa staining. (C) Imunophenotyping analysis of bone marrow cells in mice transduced with the indicated retroviral constructs. (D) Hematoxylin-eosin-stained spleen and thymus sections showing massive leukemic infiltration in AML1/ETO+KRAS ${ }^{G 12 D} /$ AML1/ETO+NRAS ${ }^{G 12 D}$ mice. Bars, $50 \mu \mathrm{m}$.

were cultured in the presence or absence of polybrene and FBS. The viral titers generated by transfection of the inducible packaging plasmids were $1.125 \times 10^{8} \mathrm{TU} / \mathrm{mL}$ for $\mathrm{pCDH}-$ KRAS $^{G 12 D}, 1.1 \times 10^{8} \mathrm{TU} / \mathrm{mL}$ for $\mathrm{pCDH}-\mathrm{NRAS}{ }^{G 12 D}$ and $1.0 \times 10^{8} \mathrm{TU} / \mathrm{mL}$ for pCDH-AML1/ETO. As shown in Figure 1B, when the pCDH-KRAS ${ }^{G 12 D}$ vector or pCDH-NRAS ${ }^{G 12 D}$ vector were transduced into mouse bone marrow cells, more than $90 \%$ of the resulting cell populations were GFP positive (GFP+) under the fluorescence microscope.

\section{Co-transduction of K-ras G12D and AML1/ETO induces acute monoblastic leukemia}

Mouse bone marrow cells were isolated, transformed with the viral vectors, transplanted into irradiated mice, and the effect of the RAS mutations and AML1/ETO translocation on the survival rate of the mice was investigated. No mortalities were observed in the mice transplanted with NRAS-transformed cells or KRAS-transformed cells up to 209 days, while the mice that were transplanted with AML1/ETO + NRAS co-transduced bone marrow cells had the highest mortality rate (95\%). Mice transplanted with AML1/ETO-transduced cells or KRAS-transduced cells had a lower mortality rate than mice transplanted with AML1/ ETO + KRAS co-transduced cells (median survival, AML1/ETO + KRAS vs. KRAS, 115 vs. 150 d, $P<0.0001 ; A M L 1 / E T O+K R A S$ vs. AML1/ETO, 115 vs. 150 d, $P<0.0001 ;$ Fig. 2A). 
A

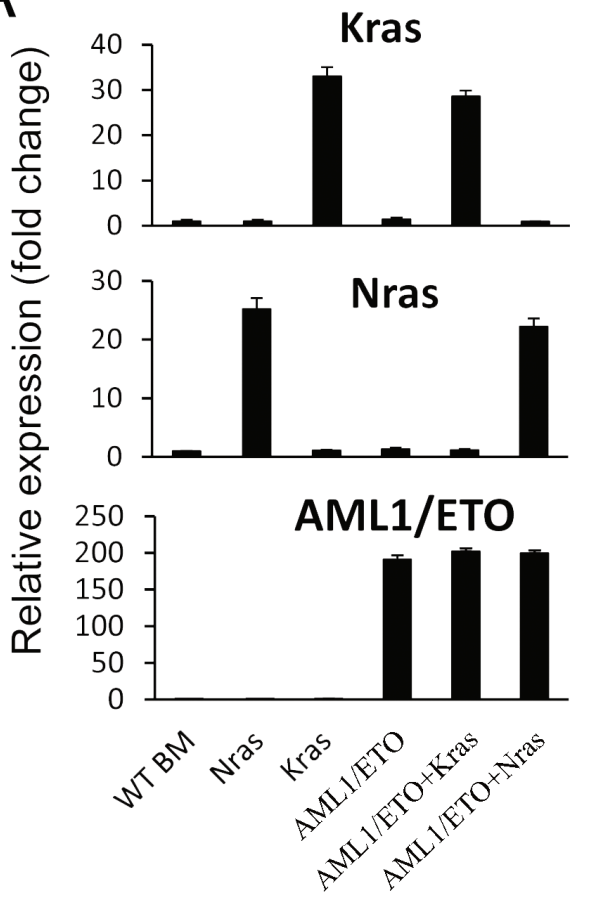

$\mathrm{B}$

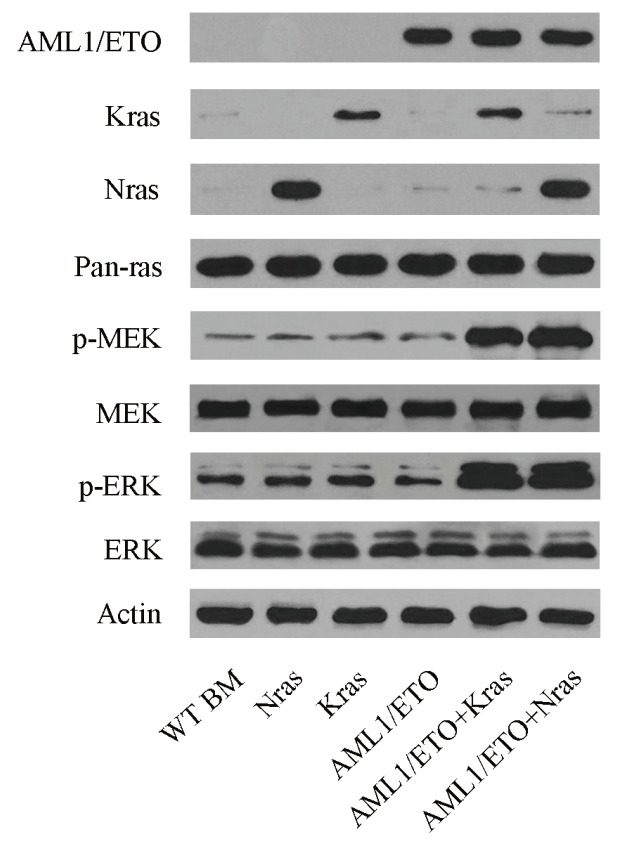

Fig. 4. Expression analysis of retroviral oncogenes in the bone marrow cells of the transplanted mice. (A) Qualitative and quantitative PCR analysis of NRAS, KRAS and AML1/ETO transcript expression in the bone marrow cells of mice transduced with the indicated retroviral constructs. (B) Western blotting of N-ras, Kras, AML1/ETO, Pan-ras, MEK, p-MEK, ERK, p-ERK and $\beta$-actin protein expression in the bone marrow cells of mice transduced with the indicated retroviral constructs.

In human patients, AML is characterized by the appearance of malignant myeloid progenitors, which rapidly accumulate in the bone marrow and aggressively infiltrate extramedullary tissues [25]. When the mice transplanted with bone marrow cells transduced with $A M L 1 / E T O+K R A S$ or $A M L 1 / E T O+N R A S$ reached terminal disease stage, EGFP-positive cells dominated the spleen (59.79\% for AML1/ETO + KRAS, 72.38\% for AML1/ETO + NRAS), lymph nodes (52.80\% for AML1/ETO + KRAS, 43.05\% for AML1/ETO + NRAS), peripheral blood (97.23\% for AML1/ETO + KRAS, $98.08 \%$ for AML1/ETO + NRAS) and central nervous system tissue (CNS; $5.78 \%$ for AML1/ETO + KRAS, 10.61\% for AML1/ETO + NRAS; Fig. 2B).

We also investigated the composition of the bone marrow cells in the indicated transplanted mice by FACS. The results showed that the bone marrow cells transduced with K-ras ${ }^{\mathrm{G} 12 \mathrm{D}}+\mathrm{AML1} / \mathrm{ETO}$ predominantly contained immature myeloid precursors $\left(\mathrm{EGFP}^{+} / \mathrm{c}-\right.$ $\mathrm{Kit}^{+} / \mathrm{Mac}-1^{-} / \mathrm{Gr}-1^{-}$cells; Fig. 3C). Wright-Giemsa staining of the peripheral blood cells and bone marrow cells of mice from the AML1/ETO + KRAS group and AML1/ETO + NRAS group confirmed the presence of morphologically-identifiable leukemia cells, characterized by progressive anemia, leukocytosis, abnormal nuclei and cytoplasmic vacuoles, in the peripheral blood and bone marrow (Fig. $3 \mathrm{~A}$ and B). Histologic analyses revealed that in mice transplanted with AML1/ETO + KRAS or AML1/ETO + NRAS co-transduced cells, massive leukemic infiltrations were closely packed in dense sheets that effaced the normal architecture of spleen and thymus in mice (Fig. 3D).

We then examined the mRNA and protein expression of $K R A S / \mathrm{K}$-ras, $N R A S / \mathrm{N}$-ras and AML1/ETO in the bone marrow cells of the transplanted mice. The levels of KRAS mRNA increased in the bone marrow cells of the K-ras group and AML1/ETO + K-ras group (Fig. $4 \mathrm{~A}$ ). K-ras protein expression was only upregulated in the bone marrow cells of the K-ras group and AML1/ETO + K-ras group (Fig. 4B). A similar trend in the expression of N-ras 


\section{Cellular Physiology and Biochemistry}

Cell Physiol Biochem 2014;33:78-87

DOI: 10.1159/000356651

Published online: January 17, 2014

c) 2014 S. Karger AG, Basel

www.karger.com/cpb

85

Zhao et al.: Development of a Mouse Model Mimicking Human AML

was observed in the N-ras group and AML1/ETO + N-ras group (Fig. 4A and B). Overall Ras levels (pan-Ras) did not show significant elevation. As a downstream Ras effector in the MAPK cascades, the phosphorylation of MEK/ERK was significantly enhanced in AML1/ETO + K-ras and AML1/ETO + N-ras group (Fig. 4B).

\section{Discussion}

Vectors based on lentiviruses show great promise as gene transfer vehicles due to their ability to transduce non-dividing cells [27]. Therefore, we constructed three lentiviral expression vectors (pCDH-K-ras ${ }^{G 12 D}$, pCDH-N-ras ${ }^{G 12 D}$ and pCDH-AML1/ETO), which produced a high viral titer after infection of $293 \mathrm{~T}$ cells $\left(>10^{8} \mathrm{TU} / \mathrm{mL}\right)$. Accurate measurements of the physical and infectious titers of lentiviral vectors are crucial for evaluating efficacy in preclinical studies and for determining the safety and efficacy in clinical studies [28]. The ability of lentiviral vectors to infect 293T cells in vitro is not always indicative of the rates of infection for other types of cells. However, when mouse bone marrow cells were transduced with the pCDH-K-ras ${ }^{\mathrm{G} 12 \mathrm{D}}$ vector or $\mathrm{pCDH}-\mathrm{N}-\mathrm{ras}^{\mathrm{G} 12 \mathrm{D}}$ vector, the frequency of the $\mathrm{EGFP}^{+}$populations was $>90 \%$. This demonstrates that co-infection of bone marrow cells with pCDH-K-ras ${ }^{G 12 D}$ or pCDH-N-ras ${ }^{G 12 D}$ and pCDH-AML1/ETO was reliable, sensitive, and reproducible.

Acute myeloid leukemia (AML) is a malignant disorder of the progenitor cells involved in myeloid hematopoiesis and exemplifies a genetically heterogeneous cancer. More than 100 genetic aberrations have been reported to be implicated in this disease [25]. It is thought that the emergence of AML requires cooperation between defects in myeloid differentiation and the presence of pro-proliferative mutations, typically involving the Ras signaling pathways [29]. N-ras (G12D) can cooperate with both AML1/ETO and MLL/ENL to accelerate the onset of leukemia and reduce overall survival in mouse models [25]. Our study confirmed that $\mathrm{N}-$ ras(G12D) was not oncogenic on its own; however, N-ras (G12D) cooperated with AML1/ ETO to accelerate disease progression compared with AML1/ETO alone (median survival 79 d vs. 150 d, $P<0.0001$; Fig. 2 A). The mammalian Ras subfamily proteins (H-Ras, N-Ras and K-Ras) are highly conserved across different species and play functionally significant roles in numerous cellular processes. Furthermore, mutations in all three Ras isoforms may occur within the same tumor in some thyroid adenomas and carcinomas, suggesting that each isoform may contribute to different aspects of tumor growth [30]. Several animal models have also been generated to study the role of the K-ras mutation in tumorigenesis [1, 15-17]. For example, an animal model expressing the oncogenic $\mathrm{K}-\mathrm{ras}^{\mathrm{LA}}$ allele was initially constructed via homologous recombination. However, K-ras ${ }^{\mathrm{LA}}$ mice develop a variety of tumor types including lung neoplasia [15]. Here we report that K-ras (G12D) cooperates with the AML1/ETO translocation to induce AML in mice (median survival, AML1/ETO + KRAS vs. KRAS, 115 vs. 150 d, $P<0.0001 ; A M L 1 / E T O+K R A S$ vs. AML1/ETO, 115 vs. 150 d, $P<0.0001$; Fig. 2A). Wright-Giemsa-stained bone marrow cytocentrifugation revealed predominantly immature blasts in AML1/ETO + N-ras leukemia, while AML1/ETO + K-ras leukemia was also characterized by immature myelomonocytic cells at various levels of differentiation (Fig. 3A and B). Additionally, bone marrow immunphenotyping of AML1/ $\mathrm{ETO}+\mathrm{K}$-ras leukemic mice demonstrated the infiltration of $\mathrm{EGFP}^{+} / \mathrm{c}-\mathrm{Kit}^{+} / \mathrm{Mac}-1^{-} / \mathrm{Gr}-1^{-}$ immature blasts. In summary, mosaic mouse models based on cooperation of K-ras with AML1/ETO can reflect the common genetic and pathologic features of human AML. This result is in support of the idea that K-ras mutations also play an important role in the pathogenesis of AML [31].

Activating mutations in the ras genes, such as the N-ras mutation, are viewed as an additional hit in AML1/ETO-rearranged leukemia [25, 32, 33]. We observed similar results in this mouse model. Mice in the AML1/ETO + N-ras (G12D) group had the lowest median overall survival rate (79 d), similar to previous reports [25]. However, our evidence suggests that enforced expression of mutant K-ras (G12D) alone was insufficient to initiate leukemia in 


\section{Cellular Physiology and Biochemistry}

Cell Physiol Biochem 2014;33:78-87

DOI: $10.1159 / 000356651$

Published online: January 17, 2014

c) 2014 S. Karger AG, Basel

www.karger.com/cpb

mice; however, expression of K-ras (G12D) was confirmed to be sufficient to induce abnormal cellular proliferation in the presence and cooperation of the AML1/ETO translocation, which acts as an anti-differentiation gene [26, 32].

In human patients, AML is characterized by the appearance of malignant myeloid progenitors, which rapidly accumulate in bone marrow and aggressively infiltrate the extramedullary tissues $[25,26]$. Histologic analyses revealed that the leukemia which developed in mice transplanted with AML1/ETO + N-ras-transduced bone marrow cells predominantly contained immature blasts. Bone marrow immunphenotyping in AML1/ETO + K-ras leukemic mice confirmed the infiltration of $\mathrm{EGFP}^{+} / \mathrm{c}^{-\mathrm{Kit}^{+}} / \mathrm{Mac}-1^{-} / \mathrm{Gr}-1^{-}$immature blasts, which parallels observations in other models of AML [25, 34, 35]. Mice harboring leukemia coexpressing AML1/ETO together with N-ras (G12D) or K-ras (G12D) displayed progressive anemia and leukocytosis. Upon reaching a terminal disease stage, EGFP-positive cells dominated the spleen, lymph nodes, peripheral blood and central CNS of mice in the AML1/ETO+K-ras group. In conclusion, we generated a mouse model of leukemia which accurately reflects the genetics and pathology of human AML. We found that K-ras ${ }^{G 12 D}$ cooperated with AML1/ETO to accelerate the onset of leukemia and reduce overall survival. These data demonstrate that mouse leukemias coexpressing AML1/ETO together with oncogenic K-ras can provide accurate yet distinct models of human AML which represent the typical clinical symptoms of different subtypes of human AML. These mouse models will be useful for studying the mechanisms by which K-ras mutations contribute to the development of myeloid leukemia, should prove useful for investigating the signaling pathways that promote self-renewal in AML, and may provide valuable tools for assessing the translational and post-transcriptional regulatory mechanisms that activate these pathways. In future research, we will investigate whether mice harboring leukemia coexpressing AML1/ETO together with N-ras (G12D) or K-ras (G12D) displaying progressive anemia and leukocytosis are regulated by the same mechanism.

\section{Acknowledgments}

This work was supported by the Shanghai Committee of Science and Technology, China(Grant No. 10140900800).

\section{References}

1 Johnson L, Mercer K, Greenbaum D: Somatic activation of the K-ras oncogene causes early onset lung cancer in mice. Nature 2001;410:1111-1116.

2 Bos JL: Ras oncogenes in human cancer: a review. Cancer Res 1989;49:4682-4689.

3 Croce CM: Oncogenes and cancer. N Engl J Med 2008;358:502-511.

4 Rodenhuis S: Ras and human tumors. Semin Cancer Biol 1992;3:241-247.

5 Marchetti A, Milella M, Felicioni L: Clinical implications of KRAS mutations in lung cancer patients treated with tyrosine kinase inhibitors: an important role for mutations in minor clones. Neoplasia 2009;11:10841092.

6 Sasaki H, Okuda K, Kawano O: Nras and Kras mutation in Japanese lung cancer patients: Genotyping analysis using LightCycler. Oncol Rep 2007;18:623-628.

7 Wang Z, Feng Y, Bardessy N, Wong KK, Liu XY, Ji H: Temporal dissection of K-ras(G12D) mutant in vitro and in vivo using a regulatable K-ras(G12D) mouse allele. PLoS One 2012;7:e37308.

-8 Zaker F, Darley RL, al Sabah A, Burnett AK: Oncogenic RAS genes impair erythroid differentiation of erythroleukaemia cells. Leuk Res 1997;21:635-640.

-9 Sabnis AJ, Cheung LS, Dail M: Oncogenic Kras initiates leukemia in hematopoietic stem cells. PLoS Biol 2009;7:e59.

10 Ahlquist T, Bottillo I, Danielsen SA: RAS signaling in colorectal carcinomas through alteration of RAS, RAF, NF1, and/or RASSF1A. Neoplasia 2008;10:680-686, 2 p following 6. 


\section{Cellular Physiology and Biochemistry}

Cell Physiol Biochem 2014;33:78-87

\begin{tabular}{l|l}
\hline DOI: $10.1159 / 000356651$ & (C) 2014 S. Karger AG, Basel
\end{tabular}

Zhao et al.: Development of a Mouse Model Mimicking Human AML

11 Liu L, Zhu S, Gong Z, Low BC: K-ras/PI3K-Akt signaling is essential for zebrafish hematopoiesis and angiogenesis. PLoS One 2008;3:e2850.

$>12$ Wells V, Downward J, Mallucci L: Functional inhibition of PI3K by the betaGBP molecule suppresses RasMAPK signalling to block cell proliferation. Oncogene 2007;26:7709-7714.

13 Menges CW, McCance DJ: Constitutive activation of the Raf-MAPK pathway causes negative feedback inhibition of Ras-PI3K-AKT and cellular arrest through the EphA2 receptor. Oncogene 2008;27:2934-2940.

14 Bos JL, Rehmann H, Wittinghofer A: GEFs and GAPs: critical elements in the control of small G proteins. Cell 2007;129:865-877.

15 Jackson EL, Willis N, Mercer K: Analysis of lung tumor initiation and progression using conditional expression of oncogenic K-ras. Genes Dev 2001;15:3243-3248.

16 Tuveson DA, Shaw AT, Willis NA: Endogenous oncogenic K-ras(G12D) stimulates proliferation and widespread neoplastic and developmental defects. Cancer Cell 2004;5:375-387.

-17 Fisher GH, Wellen SL, Klimstra D: Induction and apoptotic regression of lung adenocarcinomas by regulation of a K-Ras transgene in the presence and absence of tumor suppressor genes. Genes Dev 2001;15:3249-3262.

18 Gardini A, Cesaroni M, Luzi L: AML1/ETO oncoprotein is directed to AML1 binding regions and co-localizes with AML1 and HEB on its targets. PLoS Genet 2008;4:e1000275.

19 Li X, Xu YB, Wang Q: Leukemogenic AML1-ETO fusion protein upregulates expression of connexin 43: the role in AML 1-ETO-induced growth arrest in leukemic cells. J Cell Physiol 2006;208:594-601.

20 Yuan Y, Zhou L, Miyamoto T: AML1-ETO expression is directly involved in the development of acute myeloid leukemia in the presence of additional mutations. Proc Natl Acad Sci U S A 2001;98:10398-10403.

21 Zhang DE, Burel S, Zhou L, Hetherington CJ, Yuan Y: AML1 and AML1 fusion protein AML1-ETO in myeloid gene regulation and leukemogenesis. Blood Cells Mol Dis 2001;27:368-376.

-22 Steffen B, Knop M, Bergholz U: AML1/ETO induces self-renewal in hematopoietic progenitor cells via the Groucho-related amino-terminal AES protein. Blood 2011;117:4328-4337.

23 Kelly LM, Kutok JL, Williams IR: PML/RARalpha and FLT3-ITD induce an APL-like disease in a mouse model. Proc Natl Acad Sci USA 2002;99:8283-8288.

24 Ono R, Nakajima H, Ozaki K: Dimerization of MLL fusion proteins and FLT3 activation synergize to induce multiple-lineage leukemogenesis. J Clin Invest 2005;115:919-929.

-25 Zuber J, Radtke I, Pardee TS: Mouse models of human AML accurately predict chemotherapy response. Genes Dev 2009;23:877-889.

-26 Wang YY, Zhao LJ, Wu CF: C-KIT mutation cooperates with full-length AML1-ETO to induce acute myeloid leukemia in mice. Proc Natl Acad Sci USA 2011;108:2450-2455.

27 Kuate S, Stefanou D, Hoffmann D, Wildner O, Uberla K: Production of lentiviral vectors by transient expression of minimal packaging genes from recombinant adenoviruses. J Gene Med 2004;6:1197-1205.

28 Kay MA, Glorioso JC, Naldini L: Viral vectors for gene therapy: the art of turning infectious agents into vehicles of therapeutics. Nat Med 2001;7:33-40.

29 Gilliland DG, Jordan CT, Felix CA: The molecular basis of leukemia. Hematology Am Soc Hematol Educ Program 2004:80-97.

-30 Castellano E, Santos E: Functional specificity of ras isoforms: so similar but so different. Genes Cancer 2011;2:216-231.

-31 Parikh C, Subrahmanyam R, Ren R: Oncogenic NRAS, KRAS, and HRAS exhibit different leukemogenic potentials in mice. Cancer Res 2007;67:7139-146.

-32 Schessl C, Rawat VP, Cusan M: The AML1-ETO fusion gene and the FLT3 length mutation collaborate in inducing acute leukemia in mice. J Clin Invest 2005;115:2159-2168.

-33 Wang J, Liu Y, Li Z: Endogenous oncogenic Nras mutation promotes aberrant GM-CSF signaling in granulocytic/monocytic precursors in a murine model of chronic myelomonocytic leukemia. Blood 2010;116:5991-6002.

-34 Yan M, Burel SA, Peterson L: Deletion of an AML1-ETO C-terminal NcoR/SMRT-interacting region strongly induces leukemia development. Proc Natl Acad Sci USA 2004;101:17186-17191.

- 35 Kuo YH, Landrette SF, Heilman SA: Cbf beta-SMMHC induces distinct abnormal myeloid progenitors able to develop acute myeloid leukemia. Cancer Cell 2006;9:57-68. 